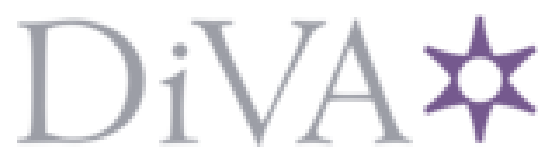

http://www.diva-portal.org

\title{
Postprint
}

This is the accepted version of a paper published in AIAA Journal. This paper has been peer-reviewed but does not include the final publisher proof-corrections or journal pagination.

Citation for the original published paper (version of record):

Bull, J., Jameson, A. (2015)

Simulation of the Taylor-Green vortex using high--order flux reconstruction schemes.

AIAA Journal, 53(9): 2750-2761

http://dx.doi.org/10.2514/1.J053766

Access to the published version may require subscription.

N.B. When citing this work, cite the original published paper.

Permanent link to this version:

http://urn.kb.se/resolve?urn=urn:nbn:se:uu:diva-264303 


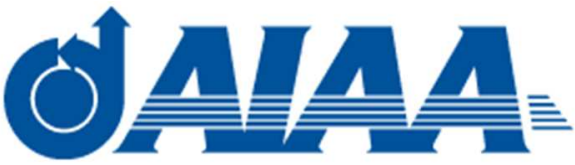

\section{Simulation of the Taylor Green Vortex using High-Order Flux Reconstruction Schemes}

\begin{tabular}{|r|l|}
\hline Journal: & AIAA Journal \\
\hline Manuscript ID: & 2014-07-J053766.R2 \\
\hline Manuscript Type: & Full Paper \\
\hline Date Submitted by the Author: & n/a \\
\hline Complete List of Authors: & $\begin{array}{l}\text { Bull, Jonathan; Stanford University, Aeronautics and Astronautics } \\
\text { Jameson, Antony; Stanford University, Aeronautics and Astronautics }\end{array}$ \\
\hline $\begin{array}{r}\text { Subject Index Category: } \\
\text { Select ONE Subject Index for } \\
\text { the Table of }\end{array}$ & $\begin{array}{l}\text { Unsteady Flows < 20000 FLUID DYNAMICS, 22100 Vortices < 20000 FLUID } \\
\text { DYNAMICS }\end{array}$ \\
$\begin{array}{r}\text { Contents. <br>This is where } \\
\text { your paper will show up in the } \\
\text { Table of Contents: }\end{array}$ & 20000 FLUID DYNAMICS \\
\hline \multicolumn{2}{|r}{} \\
\hline
\end{tabular}

SCHOLARONE

Manuscripts 


\title{
Simulation of the Taylor-Green Vortex using
}

\section{High-Order Flux Reconstruction Schemes}

\author{
J. R. Bull ${ }^{1}$ and A. Jameson ${ }^{2}$ \\ Stanford University, Stanford, CA 94305, USA
}

In this paper, we investigate the ability of high-order Flux Reconstruction (FR) numerical schemes to perform accurate and stable computations of compressible turbulent flows on coarse meshes. Two new FR schemes, which are optimized for wave dissipation and dispersion properties, are compared to the nodal Discontinuous Galerkin and Spectral Difference methods recovered via the Energy-Stable FR method. The Taylor-Green vortex benchmark problem at $R e=1600$ is used as a simple a priori test of the numerics. Dissipation rates computed from kinetic energy, vorticity and pressure dilatation are plotted against reference solutions. Results show that at low mesh resolution the FR schemes are highly accurate across a range of orders of accuracy, although oscillations can appear in the solution at orders of six and above. While the FR method has a built-in stabilization mechanism, an additional means of damping these instabilities is required. The schemes vary in the amount of numerical dissipation and resolution of the turbulent spectrum. One of the optimized FR schemes (the OFR scheme) is shown to have greater spectral accuracy than any of the others tested, motivating its future usage for high-order, high-fidelity CFD.

\footnotetext{
${ }^{1}$ Postdoctoral Scholar, Dept. of Aeronautics and Astronautics, Stanford University, AIAA Member

2 Thomas V. Jones Professor, Dept. of Aeronautics and Astronautics, Stanford University, AIAA Fellow
} 


$$
\begin{aligned}
& \Omega \quad=\text { domain } \\
& \mathbf{u}=\text { solution vector } \\
& \mathbf{f} \quad=\text { flux vector } \\
& \rho \quad=\text { density } \\
& u, v, w=\text { velocity components } \\
& \text { e } \quad=\text { energy } \\
& p \quad=\text { pressure } \\
& \xi \quad=\text { local coordinate } \\
& p \quad=\text { polynomial order } \\
& N=\text { number of solution points per direction per element } \\
& g_{L}, g_{R}=\text { left and right correction functions } \\
& l_{i} \quad=i \text { th Lagrange polynomial } \\
& L_{p} \quad=\text { degree } p \text { Legendre polynomial } \\
& c \quad=\text { free parameter in Flux Reconstruction method } \\
& J \quad=\text { Jacobian } \\
& k \quad=\text { wavenumber } \\
& E_{k} \quad=\text { kinetic energy } \\
& \zeta \quad=\text { enstrophy } \\
& \omega \quad=\text { vorticity } \\
& \mathbf{S}=\text { rate of strain tensor } \\
& \epsilon_{1}=\text { energy-based dissipation rate } \\
& \epsilon_{2}=\text { vorticity-based dissipation rate } \\
& \epsilon_{3}=\text { strain-based dissipation rate } \\
& \epsilon_{4}=\text { bulk viscosity-based dissipation rate } \\
& \epsilon_{5}=\text { dilatation-based dissipation rate }
\end{aligned}
$$




\section{Introduction}

The well-established CFD techniques of second-order numerical schemes and Reynolds-Averaged Navier Stokes (RANS) turbulence models are capable of predicting steady attached flows at cruise conditions, but they are incapable of predicting conditions at the fringes of the flight envelope which are often characterized by turbulent separated flows [1]. Many other aerodynamic problems of central importance also feature complex turbulent flows, including combustion, acoustic noise prediction and the design of hypersonic vehicles. In order to improve the performance, efficiency and safety of future generations of aircraft, CFD must move beyond the current plateau of second-order RANS methods and establish a new norm of high-order accurate, high-fidelity simulation.

High-order accurate $(p>1)$ numerical methods offer significantly better wave and vortex propagation properties than second-order accurate $(p=1)$ schemes, thanks in large part to their lower numerical dissipation. The development of high-order accurate finite difference (FD) schemes brought about new levels of accuracy in aeroacoustic problems [2], however the extension to unstructured meshes remains a major roadblock to their use for flows over and through complex geometry. Over the past two decades, Discontinuous Galerkin (DG) methods have proved to be highly successful for high-order accurate simulations in complex geometry owing to their formulation on full hybrid meshes [3, 4]. Classical DG methods construct integral operators over element volumes and surfaces by integrating the equations by parts and represent the solution within each element as a modal expansion of locally orthogonal polynomials [5]. This has the advantages of making the mass matrix diagonal and enabling exact evaluation of the integrals, but classical DG can be expensive if standard Gaussian quadrature rules are employed [6]. Nodal DG (nDG) methods, in contrast, represent the solution as nodal values at a set of interpolation points [7]. By relinquishing exact integration, nDG methods are computationally cheaper but tend to require additional stabilization $[6,7]$. The DG Spectral Element Method (DGSEM) represents the solution in classical form but performs integration cheaply using cheap quadrature formulae [8, 9]. Gassner et al. [10] devised efficient quadrature schemes for arbitrarily shaped elements based on the nodal DG approach.

Recently the collocation-based nDG method has been recast in differential form as the Spectral Difference (SD) [11, 12] and the Flux Reconstruction (FR) methods [13]. Flux Reconstruction is a 
general high-order framework which, in the case of linear fluxes, recovers particular collocation-based $\mathrm{nDG}$ and SD methods as well as allowing for the definition of new schemes [13]. The FR framework is easy to implement on unstructured meshes, allows for tuning of the numerical properties and is cheap to compute owing to the lack of integration procedures. A family of Energy-Stable Flux Reconstruction (ESFR) schemes have been developed by the Aerospace Computing Lab (ACL) at Stanford University [14]. The ESFR schemes were proven to be stable in an energy norm of Sobolev type for the 1D linear advection equation for all orders of accuracy on an arbitrary mesh [14]. Hence the ESFR schemes can be formulated on quadrilateral and hexahedral elements by taking tensor products of $1 \mathrm{D}$ operators. Subsequently the stability proof was extended to the linear advectiondiffusion equation in 1D [15], on triangles [16] and on tetrahedra [17]. The energy norm contains a non-negative coefficient $c$ which allows for the recovery of particular collocation-based nDG (with $c=0)$ and SD $\left(c=c_{S D}\right)$ schemes - henceforth denoted as FR-nDG and FR-SD resp. - as well as the $G_{2}$ scheme $\left(c=c_{G 2}\right)$ of Huynh and an infinite variety of new stable schemes [14].

For nonlinear fluxes, high-order methods are well known to be susceptible to aliasing instabilities caused by inexact representation of the true flux in a finite-dimensional polynomial subspace [7]. The aliasing error associated with the ESFR schemes arises from the use a collocation projection of the flux at the solution points [18]. It was shown for the ESFR schemes in $1 \mathrm{D}$ that the error is minimized by choosing the solution points to be the Gaussian quadrature points [18]. Recently, enhanced nonlinear stability has also been achieved in simplex elements by devising new quadrature schemes [19]. Even so, the aliasing error is still present and can become significant at higher orders of approximation, so some additional control over aliasing errors is sought. High-fidelity turbulent flow simulations which do not resolve the entire range of motions also incur a closure error (well known in the field of large eddy simulation) due to the lack of dissipation of the resolved scales by the subgrid scales. The development of methods to deal with this 'closure problem' in the context of high-order schemes is a vigorous area of research which shares much common ground with stabilization techniques.

Many techniques have been proposed for controlling aliasing and closure errors in high order numerical methods. A stabilization technique developed specifically for high-order methods is to 
apply a low-pass filter to the polynomial basis in order to reduce or remove destabilizing high wavenumber components $[9,20,21]$. An equivalent approach is to include a high-order derivative term, for example the spectral vanishing viscosity (SVV) method [22, 23]. SVV and low-pass filtering can also be used to address the closure problem, i.e. by introducing artificial dissipation to represent the effect of the subgrid scales [20, 24-28]. A technique intended solely for stabilization of high-order schemes is over-integration, also known as polynomial de-aliasing, but this incurs a high computational cost $[9,29]$. Perhaps the simplest stabilization method, commonly utilized with second-order schemes, is upwinding of the interface fluxes for example by Roe's method [30]. Upwinding with a Roe flux was shown to improve the stability of the ESFR schemes (compared to central flux) in 1D by Vincent et al. [14]. It was shown by Jameson and Lodato [31] that, for the ESFR schemes, the amount of dissipation added by the interface flux is proportional to a high-order derivative of the solution, thus providing an efficient damping of the energy in the high-order modes. The coefficient $c$ in the ESFR schemes offers another means of control over stability. Setting $c>0$ was shown to have a stabilizing effect compared to the baseline case of $c=0$ by Vincent et al. [14]. However, accuracy was reduced at the same time, implying that the choice of $c$ is a compromise between stability and accuracy. It was shown by Allaneau and Jameson [32] that a nonzero value of $c$ is identical to applying a low-pass filter to the residual in the case of a linear flux. With all of these techniques, it is vital to maintain the advantage of using high order methods over second order methods by only adding enough dissipation to stabilize the scheme - not so much that the propagation of turbulence and other wave phenomena is adversely affected.

Investigation of the spectral properties of FR schemes provides an insight into their ability to resolve multiscale phenomena such as turbulence. Vincent et al. [33] carried out a von Neumann analysis of the ESFR schemes, finding that the FR-SD method had the lowest dispersion error (they also identified a value of $c$ which maximized the allowable CFL condition). Recently, Asthana and Jameson [34] conducted a full modal analysis of the FR method in 1D to obtain dissipation and dispersion relations for each mode. They solved an optimization problem to identify a value of $c$ in the ESFR schemes that minimized errors associated with wave dissipation and dispersion; the optimized scheme is henceforth referred to as the OESFR scheme. They went further to carry 
out a multidimensional constrained optimization of the general FR method, identifying a scheme outside the ESFR family that is optimal in terms of wave propagation, henceforth referred to as the Optimized FR (OFR) scheme. We hypothesize that, by virtue of their superior resolution of the energy-containing modes, these optimized schemes will be more accurate that other FR schemes. It is shown here that the benefits of the optimized schemes will be carried to higher dimensions by taking tensor products. However, with superior accuracy comes inferior stability: the optimized schemes are more susceptible to aliasing-driven instabilities. Control over aliasing errors in FR schemes is a topic of current research within the ACL.

In this paper, we analyze the behavior of the Flux Reconstruction method in under-resolved compressible turbulent flow. The Taylor-Green Vortex (TGV) benchmark problem at $R e=1600$ is an ideal test case due to the deterministic nature of the flow, yet it contains many of the features of real turbulent flows including vortex stretching and interaction as well as dilatation (compressibility) effects. It has been used by many authors for high-order method validation including Beck and Gassner [9], Diosady and Murman [29], Chapelier et al. [5], Don et al. [35], Johnsen et al. [36] and Carton de Wiart et al. [37]. The TGV was identified as a challenging problem for high-order methods in the 1st, 2nd and 3rd International Workshops on High-Order CFD Methods [38-40]. Bull and Jameson [41] simulated the TGV problem with the FR-SD scheme, matching high-resolution reference data on relatively coarse hexahedral and tetrahedral grids. Results using more schemes and polynomial orders are presented in this paper and new details of the ability of the FR schemes to represent compressible turbulent flows emerge. The simulations are carried out using HiFiLES, the ACL's open-source unstructured GPU-accelerated Flux Reconstruction solver. Details of the code and its verification and validation can be found in Lopez et al. [42].

It is shown that the FR method accurately predicts the mean turbulent energy cascade and the important flow structures on relatively coarse grids, thanks to the high order of accuracy and to low dissipative and dispersive errors. The stabilization provided by the FR method sufficiently damps instabilities at polynomial orders of five or less, with the amount of damping depending on the particular scheme. At higher than fifth order, all schemes display instabilities at low mesh resolution (sometimes leading to residual divergence) which require further stabilization, for example 
in the form of a filter. Current work at the ACL is directed at this important topic. The OESFR scheme developed by Asthana and Jameson [34] displays nearly identical behavior to the FR-nDG scheme. The OFR scheme [34] is as stable as, but more accurate than, the FR-SD and FR-nDG schemes. Energy spectra show that the OFR scheme provides superior resolution of the energy in the higher wavenumbers, confirming that the analysis of Asthana and Jameson [34] is applicable to the Navier-Stokes equations in three dimensions.

These results lend support to the further use of high-order FR schemes - and in particular the newly developed OFR scheme - for large eddy simulation (LES) of high Reynolds number turbulent flows. Their turbulence-resolving abilities and low numerical dissipation make them suitable for applications involving far-field propagation of vortices and waves, including aircraft noise prediction and boundary layer ingestion. Future work will include the development of de-aliasing filters to improve stability at higher orders and investigation of the suitability of the OFR scheme for more complex high Reynolds number turbulent flows.

\section{High-Order Flux Reconstruction}

\section{A. General Formulation}

The compressible Navier-Stokes equations are discretized using the high-order Flux Reconstruction scheme. We write the equations in conservative form in a $3 \mathrm{D}$ domain $\Omega$ with spatial coordinates $\mathbf{x}=\left\{x_{1}, x_{2}, x_{3}\right\}$ and time $t:$

$$
\frac{\partial \mathbf{u}}{\partial t}+\frac{\partial \mathbf{f}}{\partial \mathbf{x}}=0
$$

where $\mathbf{u}=(\rho \rho u \rho v \rho w \rho e)^{T}$ are the conservative variables and $\mathbf{f}$ is the flux. The domain is split into non-overlapping elements $\Omega_{j}$. For simplicity, we consider the one-dimensional case. Inside the element a degree $p$ polynomial, defined on a set of $N=p+1$ points, is used to represent the solution and the flux, resulting in an Nth-order accurate scheme. The Gauss-Legendre quadrature points (shown in red in Figure 1) are chosen as they were found to minimize aliasing errors for nonlinear problems [18]. Additionally, fluxes are defined at the element interfaces (shown in blue in Figure 1) to facilitate coupling to neighboring elements. 
Fig. 1 Solution and flux points (red) and interface flux points (blue) in $1 \mathrm{D}$ for $\mathrm{p}=2$.

The piecewise-continuous $p$ th-order solution polynomial $u(x)$ is defined as

$$
u(x)=\sum_{i=1}^{N} u_{i} l_{i}(x)
$$

where $u_{i}(x)$ are the nodal solution values at the solution points and $l_{i}(x)$ is a set of basis functions, in this case the Lagrange polynomials. A similar expression is used to obtain the $p$ th-order flux polynomial $f(x)$. The flux polynomial in each element is extrapolated to the interfaces, giving left and right flux states $f_{L}$ and $f_{R}$ on each side of the interface. A common numerical flux $f^{*}$ is found at each interface using an approximate Riemann solver such as the Rusanov [43] or Roe method [30] for the inviscid flux and the LDG method [44] for the viscous flux. The next step is to construct a globally continuous flux polynomial. In the FR method this is achieved by adding a flux correction polynomial $\Delta f$ to the discontinuous flux $f(x)$. The correction satisfies: (a) $f+\Delta f$ equals the common interface fluxes, and (b) the corrected flux optimally represents the discontinuous flux in the element interior. $\Delta f$ is given by

$$
\Delta f(x)=\left[f_{L}^{*}-f(-1)\right] g_{L}(x)+\left[f_{R}^{*}-f(1)\right] g_{R}(x),
$$

where $f_{L}^{*}, f_{R}^{*}$ are the common interface fluxes at left and right interfaces and $g_{L}(x), g_{R}(x)$ are order $p$ polynomial correction functions satisfying $g_{L}(-1)=g_{R}(1)=1, g_{L}(1)=g_{R}(-1)=0, g_{L}(x)=$ $g_{R}(-x)$. The corrected, globally $C_{0}$-continuous flux $f^{C}$ is given by $f^{C}=f+\Delta f$. To update the solution, the divergence of the continuous flux is calculated at the solution points (first the flux is interpolated from the flux points to the solution points). An isoparametric mapping from the physical domain $x \in \Omega_{j}$ to the reference domain $\xi \in[-1,1]$ is introduced:

$$
\left.\xi\right|_{\Omega_{j}}(x)=2 \frac{x-x_{j}}{x_{j+1}-x_{j}}-1
$$

where $x_{j}, x_{j+1}$ are the end-points of the element $\Omega_{j}$. Now, denoting $\mathbf{u}_{j}^{\delta}$ as the discrete solution in element $\Omega_{j}$ and $\mathbf{f}_{j}^{\delta}$ as the discrete flux, the update step is written in semi-discrete form as

$$
\frac{\partial \mathbf{u}_{j}^{\delta}}{\partial t}=-J_{j}^{-1}\left[\mathbf{D}_{j} \mathbf{f}_{j}^{\delta}+\left(f^{*}\left(x_{j}\right)-f_{j}^{\delta}\left(x_{j}\right)\right) \mathbf{g}_{L, \xi}+\left(f^{*}\left(x_{j+1}\right)-f_{j}^{\delta}\left(x_{j+1}\right)\right) \mathbf{g}_{R, \xi}\right],
$$


where $J_{j}$ is the Jacobian in element $\Omega_{j}, \mathbf{g}_{L, \xi}$ and $\mathbf{g}_{R, \xi}$ are the derivatives of the correction functions with respect to $\xi$ at the solution points and $\mathbf{D}_{j}=\frac{\partial l_{j}}{\partial \xi}$ is the discrete derivative operator. The time derivative is discretized by an explicit fourth order Runge-Kutta scheme, thus avoiding the need to construct and invert large matrices.

\section{B. Energy-Stable Flux Reconstruction Schemes}

We consider the 1D conservation equation:

$$
\frac{\partial u}{\partial t}+\frac{\partial}{\partial x}\left(f\left(u, \frac{\partial u}{\partial x}\right)\right)=0
$$

where in general $f$ is a nonlinear function. The second-order PDE is written as a system of first-order PDEs by introducing an auxiliary variable $q$ :

$$
\begin{aligned}
\frac{\partial u}{\partial t}+\frac{\partial}{\partial x}(f(u, q)) & =0, \\
q-\frac{\partial u}{\partial x} & =0 .
\end{aligned}
$$

Now, the linear advection-diffusion equation is given by Eq. 7 with

$$
f=a u-b \frac{\partial u}{\partial x}
$$

where $a$ and $b$ are constant scalars and $b>0$. It was proven by Vincent et al. [15] that the FR schemes are energy-stable for the linear advection-diffusion equation (Eq. 8) using an 'energy method', as was used to prove stability of the linear advection problem [14, 45]. The schemes are energy-stable if the following inequality is satisfied:

$$
\frac{1}{2} \frac{d}{d t}\|U\|_{p, c}^{2}+b\|Q\|_{p, \kappa}^{2} \leq 0
$$

where $\|U\|_{p, c}$ and $\|Q\|_{p, \kappa}$ are broken Sobolev norms of the solution $u$ and the auxiliary variable $q$ which are defined as follows:

$$
\begin{aligned}
\|U\|_{p, c} & =\left\{\sum_{n=1}^{N} \int_{x_{n}}^{x_{n+1}}\left[\left(u_{n}\right)^{2}+\frac{c}{2}\left(J_{n}\right)^{2 p}\left(\frac{d u_{n}}{d x}\right)^{2}\right] d x\right\}^{1 / 2}, \\
\|Q\|_{p, \kappa} & =\left\{\sum_{n=1}^{N} \int_{x_{n}}^{x_{n+1}}\left[\left(q_{n}\right)^{2}+\frac{\kappa}{2}\left(J_{n}\right)^{2 p}\left(\frac{d q_{n}}{d x}\right)^{2}\right] d x\right\}^{1 / 2} .
\end{aligned}
$$

Here, the constants $c$ and $\kappa$ parameterize the schemes. For $c \geq 0$ and $\kappa \geq 0,\|U\|_{p, c}$ and $\|Q\|_{p, \kappa}$ are norms, and the schemes are guaranteed to be stable in accordance with Eq. 9. The proof of stability 
is quite general, as it ensures boundedness of the solution for all orders of accuracy, independent of the location of solution points within the $1 \mathrm{D}$ element. It can then be shown that to satisfy the stability condition, the correction functions $g_{L}$ and $g_{R}$ are given by

$$
\begin{aligned}
& g_{L}=\frac{(-1)^{p}}{2}\left[L_{p}-\left(\frac{\eta_{p}(c) L_{p-1}+L_{p+1}}{1+\eta_{p}(c)}\right)\right], \\
& g_{R}=\frac{1}{2}\left[L_{p}-\left(\frac{\eta_{p}(c) L_{p-1}+L_{p+1}}{1+\eta_{p}(c)}\right)\right]
\end{aligned}
$$

where $L_{p}$ is the degree $p$ Legendre polynomial, $\left.\eta_{p}(c)=\left(c(2 p+1)\left(a_{p} p !\right)\right)^{2}\right) / 2$ and $0 \leq c \leq \infty$ is the stability parameter in Eq. 10. If $c=c_{n D G}=0$, then $\eta_{p}=0$, implying

$$
\begin{aligned}
& g_{L}=\frac{(-1)^{p}}{2}\left(L_{p}-L_{p+1}\right), \\
& g_{R}=\frac{1}{2}\left(L_{p}+L_{p+1}\right),
\end{aligned}
$$

which are the left and right Radau polynomials respectively, hence $c=0$ recovers a particular FRnDG scheme as shown by Huynh [13]. The recovered scheme uses a collocation projection of the flux onto a polynomial space of degree $p$, which has significant implications for the nonlinear stability. The spectral difference (FR-SD) scheme can be recovered (for a linear flux) if the flux correction $\Delta f$ is zero at a set of $p$ points in the interior of the standard element [14]. The only way to satisfy this requirement is if $c=c_{S D}$ where $c_{S D}$ is given by

$$
c_{S D}=\frac{2 p}{(2 p+1)(p+1)\left(a_{p} p !\right)^{2}} .
$$

A third scheme, identified by Huynh as being particularly stable, is referred to as the $G_{2}$ scheme [13] and is recovered by choosing $c=c_{G 2}$ given by

$$
c_{G 2}=\frac{2(p+1)}{p(2 p+1)\left(a_{p} p !\right)^{2}} .
$$

In the general case of a nonlinear flux, it is well documented that high-order schemes suffer from aliasing-driven instabilities resulting from the projection of the flux onto a polynomial space of finite $(p)$ dimensions [7]. It was shown by Allaneau and Jameson [32] that setting $c>0$ in the ESFR schemes corresponds to damping of the highest-order polynomial mode by the application of a filter to the residual. In fact, filtering is a commonly used stabilization technique with $\mathrm{nDG}$ schemes $[7$, 21, 46]. Therefore, the FR formulation implicitly includes a stabilization mechanism. Furthermore, 
Jameson, Vincent and Castonguay [18] showed that the aliasing error associated with the ESFR schemes could be minimized in 1D by choosing the solution points to be the Gaussian quadrature points. Williams, Castonguay, Vincent and Jameson [19] devised new quadrature schemes in order to enhance nonlinear stability in simplex elements (triangles and tetrahedra).

\section{Spectral Properties}

Vincent et al. [33] performed a von Neumann analysis of the ESFR formulation, identifying dissipation and dispersion relations and calculating the order of accuracy as a function of $c$. Accuracy in this sense is correlated with the fraction of resolvable wavenumbers for which waves are propagated with negligible dissipation and dispersion. Their analysis is summarized here. Consider the 1D linear advection equation, Eq. 6 with $f=a u$, where $a$ is the advection speed, in coordinates $x^{\prime}, t^{\prime}$. Let the grid be of uniform resolution $h$ and non-dimensionalize the equation with $x=x^{\prime} / h$ and $t=t^{\prime} a / h$, so that we have

$$
\frac{\partial u}{\partial t}+\frac{\partial u}{\partial x}=0
$$

Since we know the direction of information propagation we can fully upwind the flux, i.e. $f^{*}\left(x_{j}\right)=$ $u^{\delta}\left(x_{j-1}\right)$. Then the update step Eq. 5 is rewritten as

$$
\begin{array}{r}
\frac{\partial \mathbf{u}_{j}^{\delta}}{\partial t}=-J_{j}^{-1}\left[\mathbf{C}_{0} \mathbf{u}_{j}^{\delta}+\mathbf{C}_{-1} \mathbf{u}_{j-1}^{\delta}\right] \\
\mathbf{C}_{0}=\mathbf{D}-\mathbf{g}_{L, \xi} \mathbf{l}_{L}^{T}, \\
\mathbf{C}_{-1}=\mathbf{g}_{L, \xi} \mathbf{l}_{R}^{T},
\end{array}
$$

where $\mathbf{g}_{L, \xi}$ is the gradient of the left correction function and $\mathbf{l}_{L}$ and $\mathbf{l}_{R}$ are vectors containing the values of the Lagrange polynomials on the left and right interfaces. An initial condition $u(x, 0)=e^{i k x}$ is specified which admits a solution of the form $u(x, t)=e^{i k(x-t)}$, where $k$ is the wavenumber. The solution can be expressed in the parent domain using the mapping in Eq. 4:

$$
u\left(x \in \Omega_{j}, t\right)=e^{i k(j-t)} e^{i k \frac{(\xi+1)}{2}},
$$

This infinite-dimensional exact solution must be projected to the finite-dimensional polynomial space to obtain the numerical solution:

$$
\mathbf{u}_{j}^{\delta}(t)=e^{i k\left(j-a^{\delta}(k) t\right)} \mathbf{v}
$$


where $a^{\delta}(k)$ is the numerical wavespeed as a function of wavenumber and $\mathbf{v}$ is the unknown vector associated with the projection. By introducing this numerical solution into the update step Eq. 5 we arrive at the semi-discrete dispersion relation

$$
\begin{array}{r}
\mathbf{M} \mathbf{v}=a^{\delta} \mathbf{v}, \\
\mathbf{M}=\frac{-2 i}{k}\left(\mathbf{C}_{0}+e^{-i k} \mathbf{C}_{-1}\right),
\end{array}
$$

which is a $p+1$-dimensional eigenvalue problem for each wavenumber $k$. The solution of Eq. 19 provides $p+1$ numerical modes for each $k$ with the complex eigenvalues

$$
a_{p}^{\delta}(k)=a_{p_{r}}^{\delta}(k)+i a_{p_{i}}^{\delta}(k), p=1,2, \ldots, p+1,
$$

where $a_{p_{r}}^{\delta}$ and $a_{p_{i}}^{\delta}$ are the real and imaginary numerical wavespeeds respectively. The analytical solution has the exact relation $a_{r}=1, a_{i}=0$, therefore the errors associated with numerical dispersion and dissipation are $e^{i k\left(1-a_{p_{r}}^{\delta} t\right)}$ and $e^{i k a_{p_{i}}^{\delta} t}$ respectively. The usual interpretation of the existence of multiple numerical modes for each wavenumber is that one mode is 'physical' in the sense that it most closely follows the analytical mode, while the remaining $p$ of the $p+1$ admissible modes are 'spurious'. These spurious modes are often neglected by assuming that they contain only a small fraction of the energy and are fairly dissipative [33]. The reader is referred to Asthana and Jameson [34] for a comprehensive discussion of the spurious modes.

Figure 2 plots the real and imaginary components of the physical mode against the normalized wavenumber $k /(p+1)$ for the FR-nDG scheme for polynomial orders from one to five $\left(p_{1}\right.$ to $\left.p_{5}\right)$. The real part is plotted as the effective wavenumber $k_{e f f}=a_{r}^{\delta} k /(p+1)$ and the imaginary part $a_{p_{i}}^{\delta}$ is plotted directly. The components of exact wavespeed, $a_{r}$ and $a_{i}$, are plotted for reference. As $p$ is increased, the exact solution is approximately followed over a larger proportion of the range of resolvable wavenumbers. However, the overshoot in the dispersion relation becomes more pronounced, implying a lower CFL limit. Dissipation is reduced at higher $p$, which translates as better resolving efficiency. Figure 3 plots the real and imaginary components of the physical mode for FR-nDG, FR-SD and Huynh's $G_{2}$ scheme [13] at order $p_{3}$. The effect of changing $c$ from $c=c_{n D G}=0$ to $c=c_{S D}$ is that the numerical wavespeed remains closer to the exact wavespeed for longer, but the dissipation starts increasing (i.e. $a_{p_{i}}^{\delta}<0$ ) at a lower normalized wavenumber, 
1

2

3

4

5

6

7

8

9

10

11

12

13

14

15

16

17

18

19

20

21

22

23

24

25

26

27

28

29

30

31

32

33

34

35

36

37

38

39

40

41

42

43

44

45

46

47

48

49

50

51

52

53

54

55

56

57

58

59

60

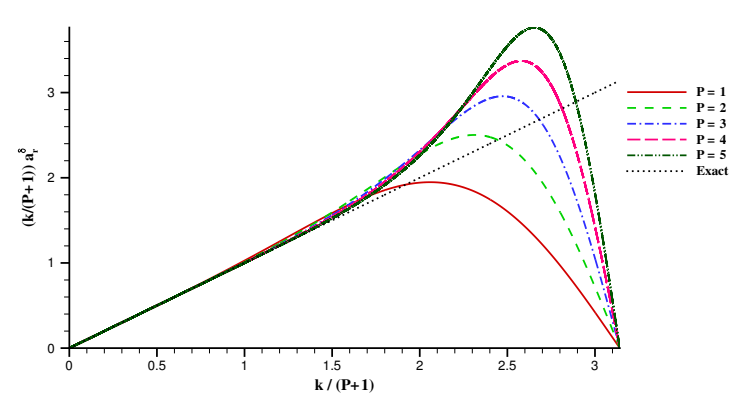

(a)

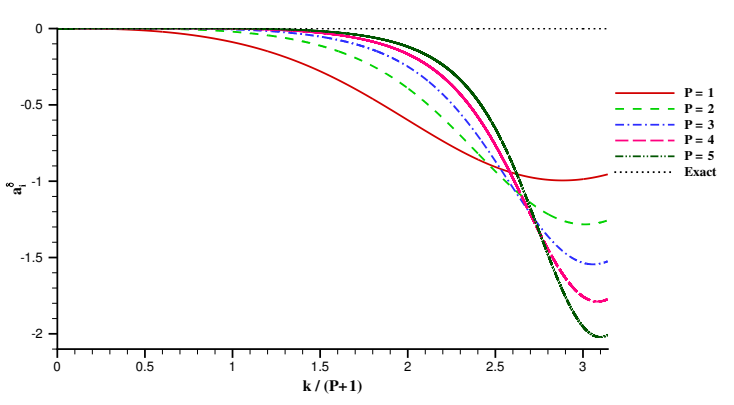

(b)

Fig. 2 Effect of polynomial order on (a) effective wavenumber $k_{e f f}$ and (b) imaginary part $a_{i}^{\delta}$ for the physical mode of the FR-nDG scheme for $p_{1}$ to $p_{5}$ (from Asthana and Jameson [34]) suggesting that an optimal scheme might exist in between FR-nDG and FR-SD. The $G_{2}$ scheme is inferior to FR-SD in terms of both errors.

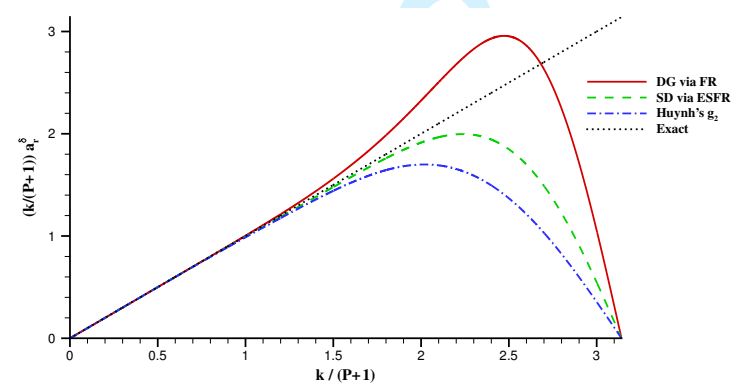

(a)

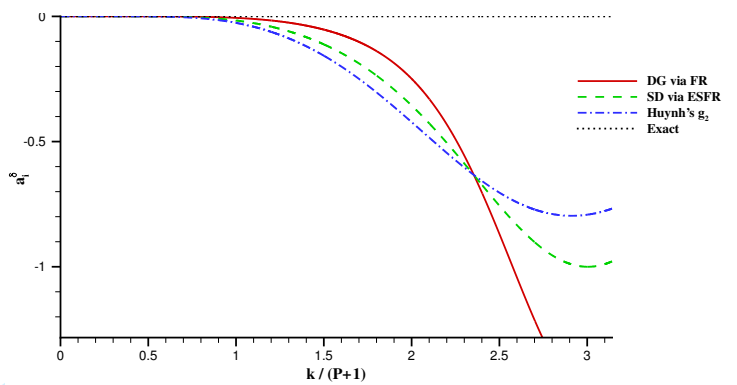

(b)

Fig. 3 (a) effective wavenumber $k_{e f f}$ and (b) imaginary part $a_{i}^{\delta}$ for the physical mode of the FR-nDG, FR-SD and $G_{2}$ schemes at order $p_{3}$ (from Asthana and Jameson [34])

\section{Optimized FR Schemes}

Recently, Asthana and Jameson [34] carried out an optimization of the ESFR schemes in spectral space using $c$ as the free parameter. They identified an optimal value of $c$ at each order $p$ for which the dissipation and dispersion errors were minimized over the range of resolvable wavenumbers, denoting the scheme as the OESFR (Optimal ESFR) scheme. Optimizing with respect to both errors balanced the competing effects described above, finding a minimum close to $c=c_{n D G}=0$. They then tackled the more complex multidimensional optimization problem of finding the zeros of the correction functions which minimized the dissipation and dispersion errors. A general form of 
the left correction function was considered:

$$
g_{L}(\xi)=\Pi_{n=1}^{p} \frac{\xi-\xi_{n}}{1+\xi_{n}} \frac{\xi-1}{2},
$$

which ensured a unity value on the left interface; the right correction function $g_{R}$ is simply a mirror of $g_{L}$. The $p$-dimensional solution space of free zeros $\left\{\xi_{1}, \xi_{2}, \ldots, \xi_{p+1}\right\}$ contains the family of ESFR schemes as a subspace. The so-called Optimal FR (OFR) schemes could then be identified subject to the constraint that they are linearly stable. For $p=1$ the OESFR scheme was recovered owing to the single degree of freedom, but for $p>1$ the schemes were outside the ESFR family. Figure 4 plots the dispersion and dissipation relations for FR-nDG, OESFR and OFR for $p_{4}$. The OESFR scheme has a slightly lower dispersion error than FR-nDG and an almost identical dissipation error, while for the OFR scheme both errors are significantly lower than FR-nDG.

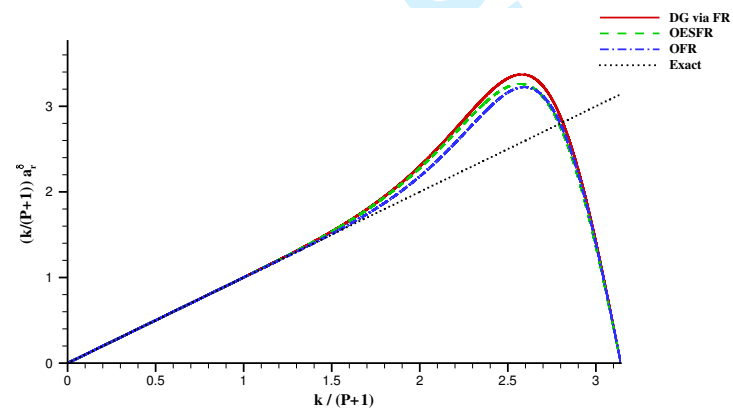

(a)

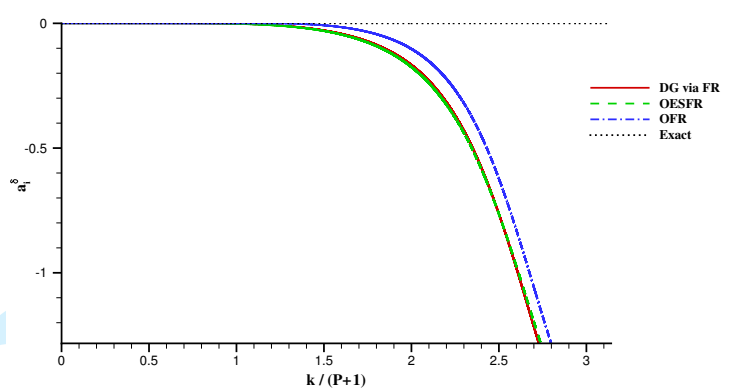

(b)

Fig. 4 (a) effective wavenumber $k_{e f f}$ and (b) dissipation error $a_{i}$ for the physical mode of the FR-nDG, OESFR and OFR schemes for $p_{4}$. From Asthana and Jameson [34]

\section{E. FR Schemes for Turbulent Flow Simulations}

The ESFR schemes have been used successfully for implicit LES of a number of challenging turbulent flows, including transitional flow over an SD7003 airfoil at $R e_{C}=60,000$ [47], transitional flow over a pitching and plunging NACA 0012 wing section [48] and unsteady flow over a flapping wing-fuselage configuration [49]. Closure of the under-resolved Navier-Stokes equations was handled in these cases by numerical dissipation emanating from the upwinded interface fluxes. Equipped with an LES model, they have also been used to accurately simulate the turbulent flow over a square cylinder at $R e=21,400$ on relatively coarse hexahedral [50] and tetrahedral [41] meshes. 
Nevertheless, there remain open questions about the schemes' behavior in under-resolved turbulent flows. It is not known which are the most accurate schemes in terms of faithfully capturing as much of the turbulent spectrum as possible when the grid resolution is larger than the Kolmogorov lengthscale. Unfortunately, it is likely that the most accurate scheme will not be the most stable, and so the question needs to be rephrased as 'which scheme has the best balance of accuracy and stability?' The results presented in the previous section encourage the use of the newly developed OFR scheme for turbulent flow simulations, where the improvements in spectral accuracy over FRnDG and FR-SD should be visible, particularly on coarse meshes where the full spectrum can not be fully resolved. It is also important to examine the role of polynomial order in simulations of turbulent flows. In order to take full advantage of high-order schemes over second order schemes including computational efficiency, we wish to use a high polynomial order. The above spectral results imply that higher orders provide better spectral resolution and lower numerical dissipation. Yet as the order is increased, it was found that aliasing instabilities grew large in simulations of the Taylor-Green Vortex [41]. Furthermore, the reduced CFL limits at higher orders might reduce the computational efficiency due to the resulting longer simulation times.

\section{The Taylor-Green Vortex}

The Taylor-Green Vortex problem is a canonical flow which provides a convenient stepping stone towards simulating real flows, requiring the solution of the Navier Stokes equations in 3D at moderate Reynolds numbers. From a simple initial datum, vortex stretching mechanisms cause the flow to decay along a well-defined trajectory, generating a detailed turbulent spectrum over a period of 20 seconds. The TGV was one of the problems in the 1st, 2nd and 3rd International Workshops on High-Order CFD Methods [38-40]) and is considered to be a challenging test for high-order methods. Various authors have used high-order discontinuous methods on the TGV test case with excellent results, including classical DG [5, 36, 37], DGSEM [9, 29] and a preliminary study by Bull and Jameson using the FR-SD scheme [41]. Wang gives a review of the current status of high-order methods for several problems including the TGV [1]. In this paper we use the TGV case to test the ability of various FR schemes to accurately represent the turbulent spectrum and examine the 
nonlinear stability of these schemes at varying orders of approximation.

\section{A. Problem Setup}

The geometry is a triply-periodic box of dimension $0 \leq(x, y, z) \leq 2 \pi$ and the initial condition is given by the following:

$$
\begin{aligned}
u\left(t_{0}\right) & =u_{0} \sin (x / L) \cos (y / L) \cos (z / L), \\
v\left(t_{0}\right) & =-u_{0} \cos (x / L) \sin (y / L) \cos (z / L), \\
w\left(t_{0}\right) & =0, \\
p\left(t_{0}\right) & =p_{0}+\frac{\rho_{0}}{16}\left[\cos \left(\frac{2 x}{L}\right)+\cos \left(\frac{2 y}{L}\right)\right]\left[\cos \left(\frac{2 z}{L}\right)+2\right],
\end{aligned}
$$

where $L=1, u_{0}=1, \rho_{0}=1$ and $p_{0}=100$. The Mach number is set to 0.08 (consistent with the initial pressure $p_{0}$ ) and the initial temperature is $300 \mathrm{~K}$. A Reynolds number of 1600 is prescribed by adjusting the viscosity. Three meshes are used: a coarse mesh of $16^{3}$ hexahedral elements, a medium mesh $\left(32^{3}\right.$ elements $)$ and a fine mesh $\left(64^{3}\right.$ elements $)$. We compare our results to several others submitted to the 1st High-Order Workshop. A high resolution reference solution was computed by Debonis [51] using Bogey and Bailley's 13-point dispersion-relation-preserving (DRP) scheme [52] on a mesh of $512^{3}$ elements. Debonis used the same scheme on meshes of $64^{3}, 128^{3}$ and $256^{3}$ elements and we also compare our results to these. The closest published results in terms of using a similar method are those of Beck and Gassner, who used a filtered fourth-order accurate DGSEM on a $64^{3}$ element mesh [9]. These are also plotted in some figures for comparison. Energy spectra are compared to a pseudo-spectral computation on a $512^{3}$ element mesh computed by Carton de Wiart et al. [37].

\section{B. Diagnostics}

Several diagnostic quantities can be computed from the flow as it evolves in time, allowing the characteristics of the numerical scheme to be observed. Firstly, the volume-averaged kinetic energy is given by

$$
E_{k}=\frac{1}{\rho_{0} V} \int_{V} \frac{1}{2} \rho \mathbf{u} \cdot \mathbf{u} d V
$$


where $V$ is the volume. Now we can compute the rate of dissipation from the kinetic energy:

$$
\epsilon_{1}=\epsilon\left(E_{k}\right)=-\frac{d E_{k}}{d t}
$$

This quantity, $\epsilon_{1}$, is referred to as the energy-based dissipation rate. In incompressible flow, it can be shown that the dissipation rate is related to the integrated enstrophy $\zeta$ by a constant [35]:

$$
\begin{array}{r}
\epsilon_{2}=\epsilon(\zeta)=\frac{2 \mu}{\rho_{0}} \zeta, \\
\zeta=\frac{1}{\rho_{0} V} \int_{V} \frac{1}{2} \rho \boldsymbol{\omega} \cdot \boldsymbol{\omega} d V
\end{array}
$$

where $\boldsymbol{\omega}$ is the vorticity and $\mu$ is the dynamic viscosity. Since the Mach number of the TGV case is low, Eq. 28 can be assumed to hold true. We refer to $\epsilon_{2}$ as the vorticity-based dissipation. In compressible flow, the dissipation rate is given by the sum of three components, $\epsilon_{3}, \epsilon_{4}$ and $\epsilon_{5}$, given by

$$
\begin{aligned}
\epsilon_{3} & =\epsilon(\mathbf{S})=2 \frac{\mu}{\rho_{0} V} \int_{V} \mathbf{S}: \mathbf{S} d V \\
\epsilon_{4} & =\epsilon\left(\mu_{v}\right)=\frac{\mu_{v}}{\rho_{0} V} \int_{V}(\nabla \cdot \mathbf{u})^{2} d V, \\
\epsilon_{5} & =\epsilon(p)=-\frac{1}{\rho_{0} V} \int_{V} p \nabla \cdot \mathbf{u} d V,
\end{aligned}
$$

where $\mu_{v}=0$ is the bulk viscosity (thus $\epsilon_{4}$ is neglected) and $\mathbf{S}$ is the rate-of-strain tensor [35]. The pressure dilatation-based dissipation rate, $\epsilon_{5}$, drops out in the incompressible limit and is expected to be small at Mach 0.08. Therefore, the strain-based dissipation rate, $\epsilon_{3}$ is expected to be almost identical to $\epsilon_{2}$.

The vorticity-based dissipation rate is a measure of how well the vorticity-carrying small scales (i.e. the inertial range of turbulence) are resolved, and is considered to be a sensitive measure of how accurately turbulence is resolved by a numerical method. Numerical dissipation reduces the sharpness with which velocity gradients are approximated [35]. It can be estimated by the difference between $\epsilon_{1}$ and $\epsilon_{2}$ and will be nonzero for any method which is not kinetic-energy-preserving [1,37]. This is a useful error measure in that it is independent of the reference solution and could be used in more complex flows [1]. 


\section{Results}

\section{Effect of Polynomial Order}

To illustrate the effect of polynomial order on the accuracy and stability of the solution, Figure 5 shows energy-based dissipation rate $\epsilon_{1}$ and vorticity-based dissipation rate $\epsilon_{2}$ using FR-nDG (a, b), FR-SD (c, d) and OFR (e, f) at a constant 128 DoF per direction given by $p_{7}$ on the coarse, $p_{3}$ on the medium and $p_{1}$ on the fine meshes (denoted $16 \times 8,32 \times 4$ and $64 \times 2$ DoF in the Figure). The OESFR scheme obtained identical results to FR-nDG because $c_{O E S F R}$ is very close to $c_{n D G}=0$; therefore the OESFR results are not shown here. A reference $\epsilon_{2}$ solution from the high-order workshop computed by Debonis [51] using the DRP scheme on a $512^{3}$ element mesh is also plotted, denoted by 'DRP-512' (note that at this high level of resolution $\epsilon_{1}$ and $\epsilon_{2}$ are equal so it is not necessary to plot the reference $\epsilon_{1}$ solution as well). Also included in Figure $5(\mathrm{~b})$ is a reference $\epsilon_{2}$ solution with an equivalent number of DoF to the current results, namely the DRP scheme on a $128^{3}$ mesh computed by Debonis [51], denoted by 'DRP-128'.

We can observe the competing effects of stability and accuracy: the low-order $\left(p_{1}\right)$ solutions on the fine mesh are inaccurate due to excessive dissipation, while the high-order $\left(p_{7}\right)$ solutions on the coarse mesh oscillate before diverging (FR-nDG at $t \approx 12$, FR-SD at $t \approx 9$ and OFR at $t \approx 7.5)$. It is apparent that at $p_{7}$ the OFR scheme is the least stable because it diverged the earliest. The over-prediction of $\epsilon_{2}$ by this scheme is indicative of too little dissipation in the smallest resolved scales. The 'Goldilocks' solutions $-p_{3}$ on the medium mesh $(32 \times 4)$ - have a good balance of accuracy and stability. The oscillations in the $p_{7}$ solutions are thought to be caused by representing the flow structures with high order polynomials, which naturally contain large variations. In a real turbulent flow, one expects these variations to disappear when considering average quantities over a sufficiently large volume. However, the TGV flow has several symmetries so that variations occurring simultaneously in different parts of the flow may add up. These results confirm that some means of dealiasing is essential for very high order FR schemes, in particular the low-dissipation OFR scheme, when the flow is under-resolved. However, in order to preserve the accuracy of the schemes, stabilization should only be added when and where necessary to control the growth of oscillations. For example, filtering could be applied to individual elements or even solution points 
according to a shock sensor [53]. This approach is being developed for the FR schemes by Sheshadri and Jameson [54]. The results also show that $\epsilon_{2}$ is a more sensitive measure of accuracy, but $\epsilon_{1}$ is a more sensitive measure of stability.

Figure 6 plots $\epsilon_{1}$ and $\epsilon_{2}$ from the $p_{3}$ FR-SD solution on the fine mesh, denoted 'tke' and 'vort' respectively, versus the DRP-512 solution and a solution with an equivalent number of DoF, namely filtered fourth-order DGSEM on a $64^{3}$ mesh computed by Beck and Gassner [9]. The numerical dissipation of the FR scheme (denoted as 'num. diss.'), given by $\epsilon_{1}-\epsilon_{2}$, is also plotted. $\epsilon_{1}$ given by FR-SD almost exactly matches the reference solutions with no oscillations, providing further evidence that fourth-order FR schemes strike the right balance between accuracy and stability. The numerical dissipation is small, indicating that the majority of the kinetic energy loss is via molecular dissipation as observed by Beck and Gassner [9].

\section{Effect of FR Scheme}

Figures 7 (a), (c) and (e) show close-ups of the peak of $\epsilon_{1}$ computed using the FR-nDG, FRSD and OFR schemes at orders $p_{3}$ to $p_{6}$ on the medium mesh versus the DRP-128 and DRP-512 solutions. At $p_{3}$ all the schemes behave in a similar manner, under-predicting the peak. At $p_{4}$ and $p_{5}$ all the schemes capture the peak fairly well but small oscillations appear in the $p_{5}$ solutions. The $p_{6}$ solutions all display significant oscillations close to $t=9$. When the three schemes at orders $p_{3}$ to $p_{6}$ were used on the coarse mesh (results not shown), only the FR-nDG scheme did not diverge at orders $p_{5}$ and $p_{6}$, suggesting that FR-nDG is the most stable scheme (of those tested) at low resolution.

Figures 7 (b), (d) and (f) show close-ups of the peak of vorticity-based dissipation rate $\epsilon_{2}$ computed using the same schemes and orders as (a), (c) and (e). As in Figure 5, differences in accuracy between the schemes are much more visible than in the $\epsilon_{1}$ plots. FR-nDG under-predicts the peak at all orders, FR-SD under-predicts the peak slightly less, and OFR predicts the peak fairly accurately at orders greater than $p_{3}$. The $p_{6}$ OFR solution matches the reference data very well. The slight over-prediction of $\epsilon_{2}$ (i.e. vorticity strength) by the $p_{5}$ OFR scheme is suggestive of excess energy in the small resolved scales. This is observed to a greater degree in the 7 th order 


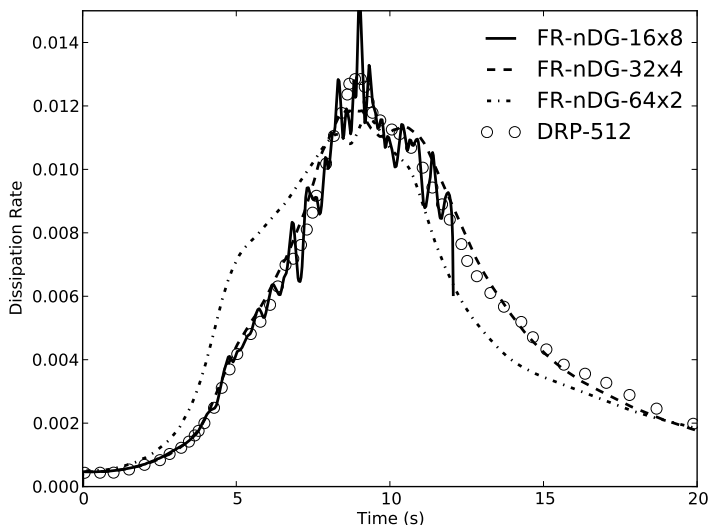

(a) $\epsilon_{1}$, FR-nDG

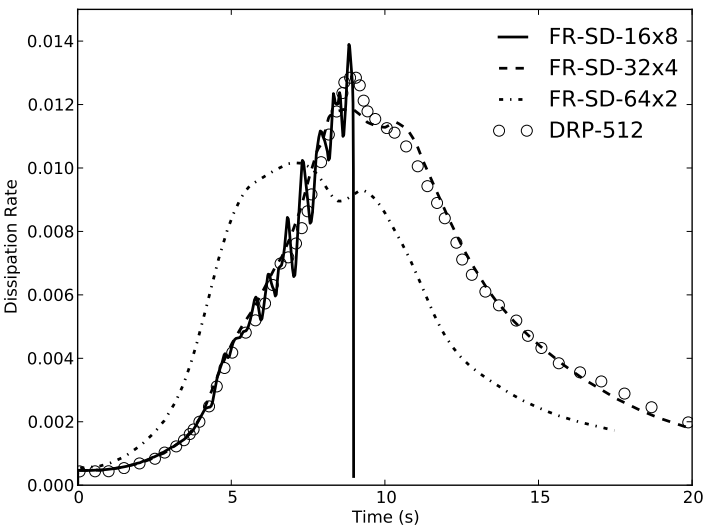

(c) $\epsilon_{1}$, FR-SD

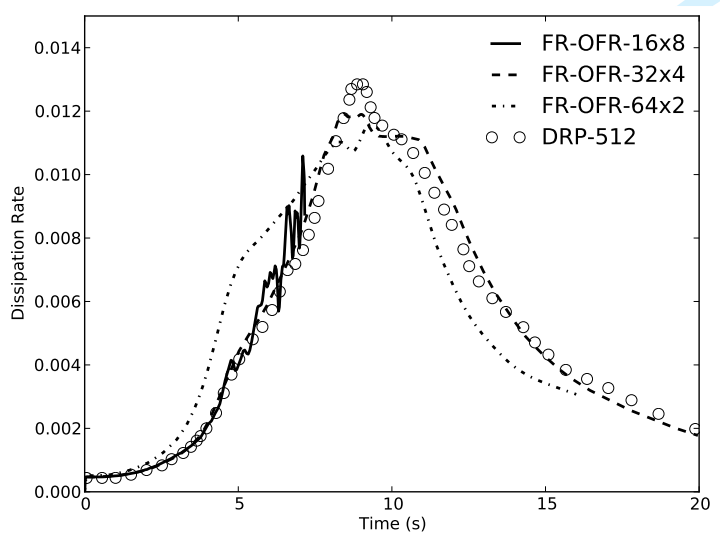

(e) $\epsilon_{1}$, OFR

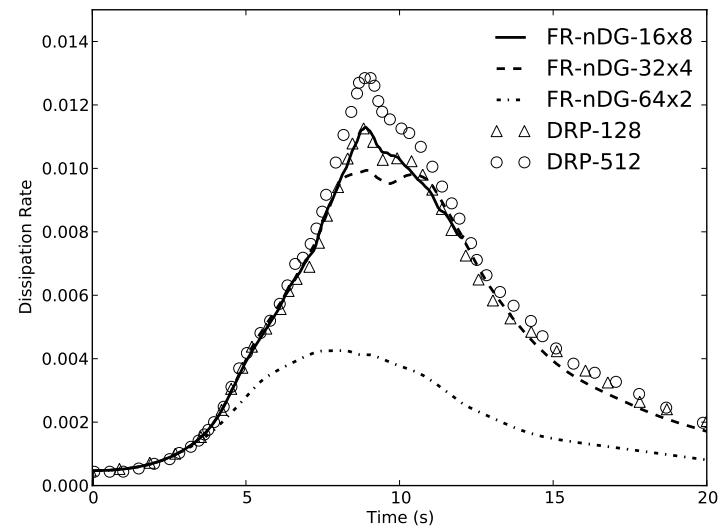

(b) $\epsilon_{2}, \mathrm{FR}-\mathrm{nDG}$

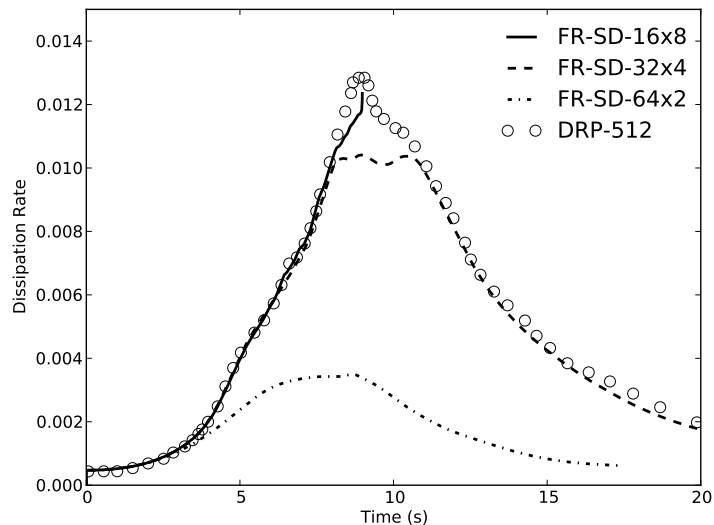

(d) $\epsilon_{2}$, FR-SD

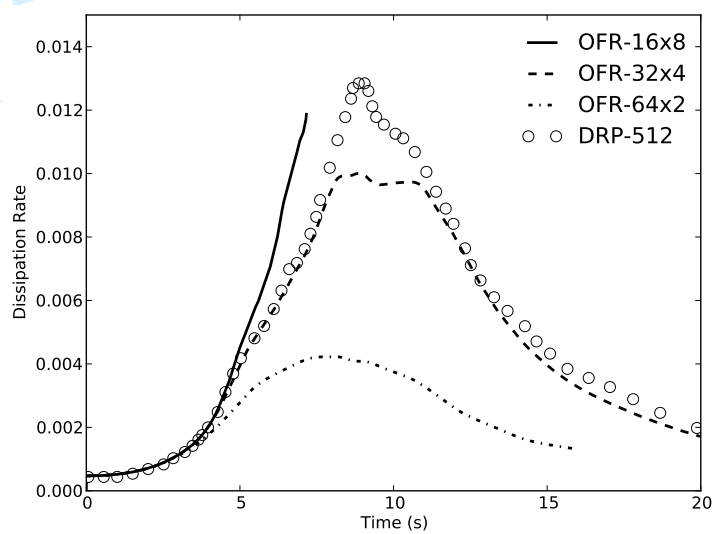

(f) $\epsilon_{2}, \mathrm{OFR}$

Fig. 5 (a, c, e) $\epsilon_{1}$ and (b, d, f) $\epsilon_{2}$ using FR-nDG, FR-SD and OFR with constant $128^{3}$ DoF $\left(p_{7}\right.$ on coarse, $p_{3}$ on medium and $p_{1}$ on fine meshes). 'Beck-DG-64x4' $=$ filtered $\mathrm{nDG}$ scheme on $64^{3}$ DoF mesh [9], 'DRP-128'/'DRP-512' = DRP scheme on $128^{3} / 512^{3}$ DoF meshes [51]. 


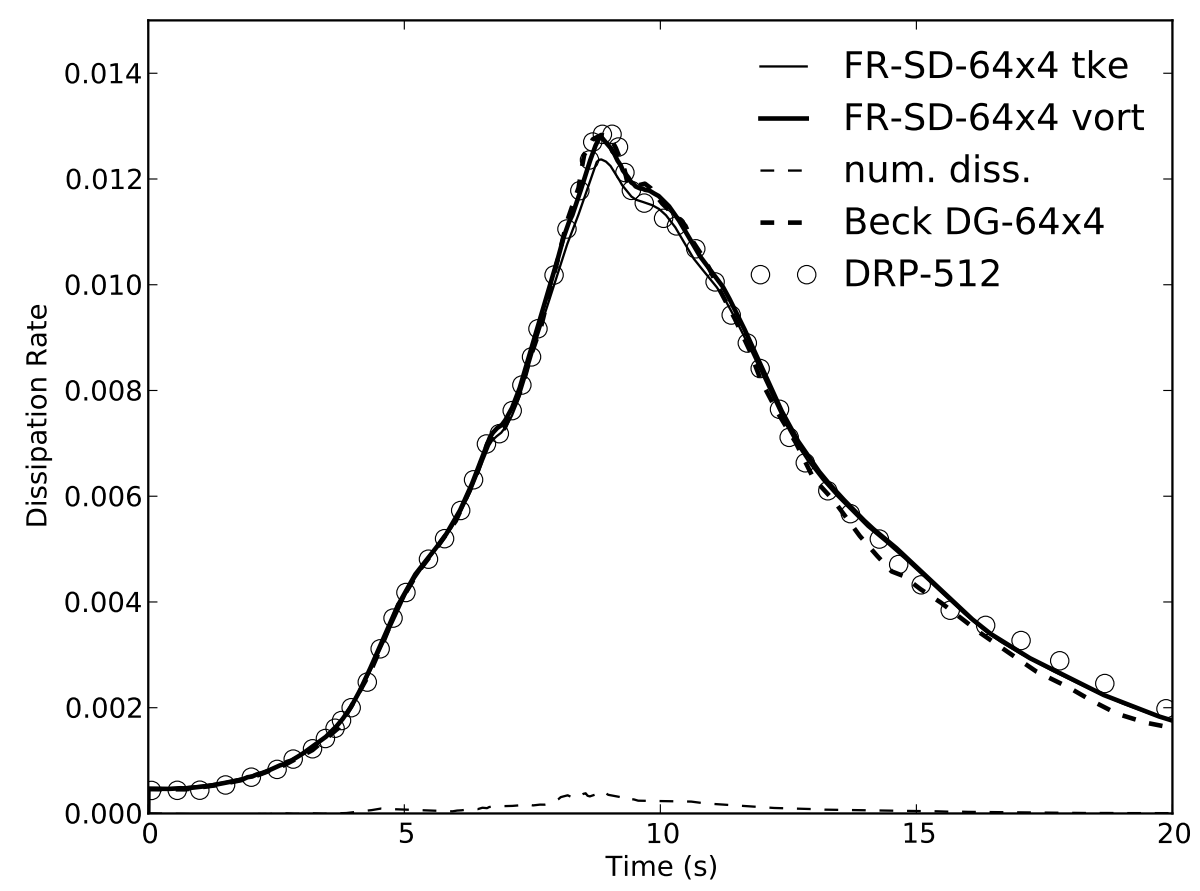

Fig. $6 \epsilon_{1}$ ('tke'), $\epsilon_{2}$ ('vort') and $\epsilon_{1}-\epsilon_{2}$ ('num. diss.') using FR-SD at $p_{3}$ on fine mesh. 'BeckDG-64x4' = filtered nDG scheme on $64^{3}$ DoF mesh [9], 'DRP-512' = DRP scheme on $512^{3}$ DoF mesh [51].

OFR solution in Figure 5 (f). In the case of a nonlinear flux it is known that the aliasing error causes energy to pile up in the scales near the grid scale due to inadequate draining by the numerical scheme or by molecular viscosity when the grid is coarse [55]. In Section II C the OFR scheme was shown to have reduced numerical dissipation compared to FR-nDG or FR-SD at high wavenumbers, implying a smaller drain of the energy and an over-prediction of vorticity. Nevertheless, these results using the new OFR scheme confirm that the theoretically superior greater accuracy is indeed borne out in practice.

\section{Compressibility Effects}

The dissipation rate due to pressure dilatation, $\epsilon_{5}$, measures the effect of compressibility on the dissipation of turbulent energy. Figure 8 plots $\epsilon_{5}$ for the FR-nDG, FR-SD and OFR schemes at $p_{4}$ on the medium mesh versus the DRP scheme on the $64^{3}$ and $512^{3}$ meshes. As the Mach number is so low, the effects of compressibility should not be very strong and $\epsilon_{5}$ is expected to be close to zero (as is the case with the DRP scheme). Note that the $y$ axis scale is stretched by a 


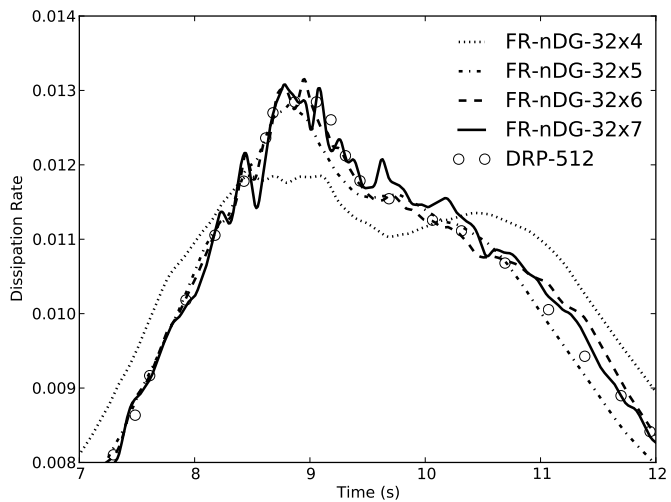

(a) $\epsilon_{1}$, FR-nDG

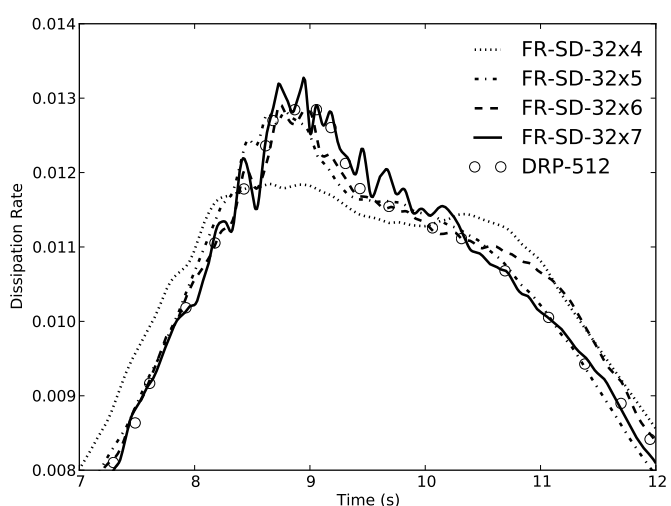

(c) $\epsilon_{1}$, FR-SD

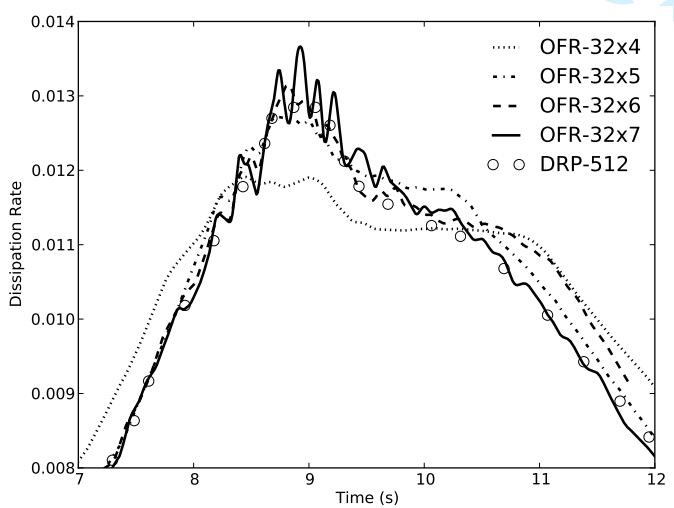

(e) $\epsilon_{1}$, OFR

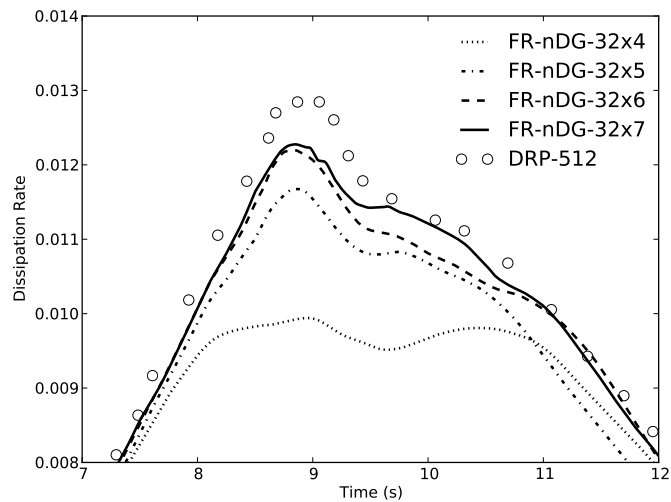

(b) $\epsilon_{2}$, FR-nDG

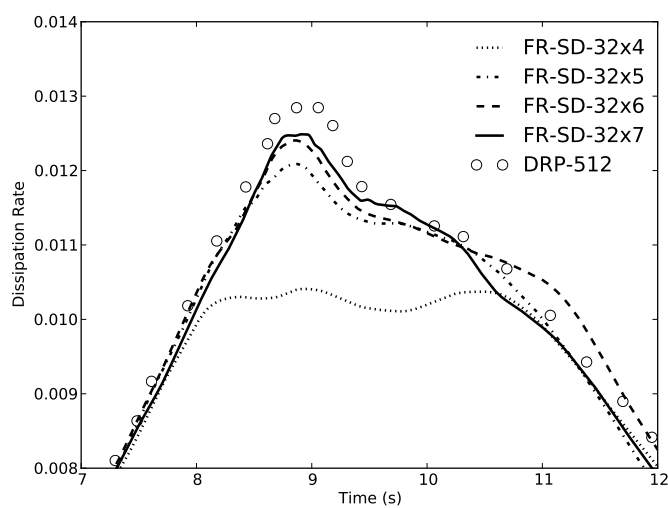

(d) $\epsilon_{2}$, FR-SD

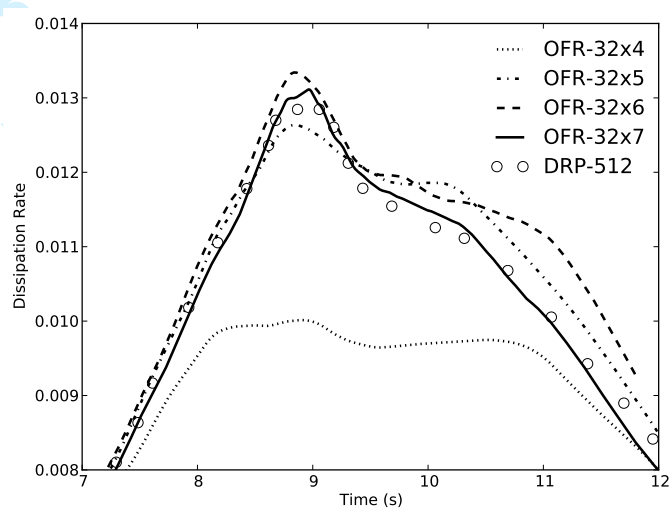

(f) $\epsilon_{2}$, OFR

Fig. 7 (a, c, e) $\epsilon_{1}$ and (b, d, f) $\epsilon_{2}$ using FR-nDG, FR-SD and OFR at $p_{3}, p_{4}, p_{5}$ and $p_{6}$ on medium mesh. 'DRP-512' = DRP scheme on $512^{3}$ DoF mesh [51].

factor of 100 compared to the previous plots. Despite having 2.5 times the number of DoF per direction, the magnitude of the variations in $\epsilon_{5}$ using FR-nDG, FR-SD and OFR are larger than those in the DRP solutions. The OFR results display the largest variations, possibly due to the 
1

lower numerical dissipation. At higher orders (not shown) it was found that all the FR schemes greatly over-predicted the magnitude of $\epsilon_{5}$, and that the sign of $\epsilon_{5}$ depended on the parity of the polynomial order. The root cause of these issues is thought to be related to the representation of the divergence term in Eq. 32 and is the subject of ongoing investigation. Nevertheless, $\epsilon_{5}$ predicted by the FR schemes was found to converge to the correct solution with grid refinement, as was also observed by Chapelier et al. [5].

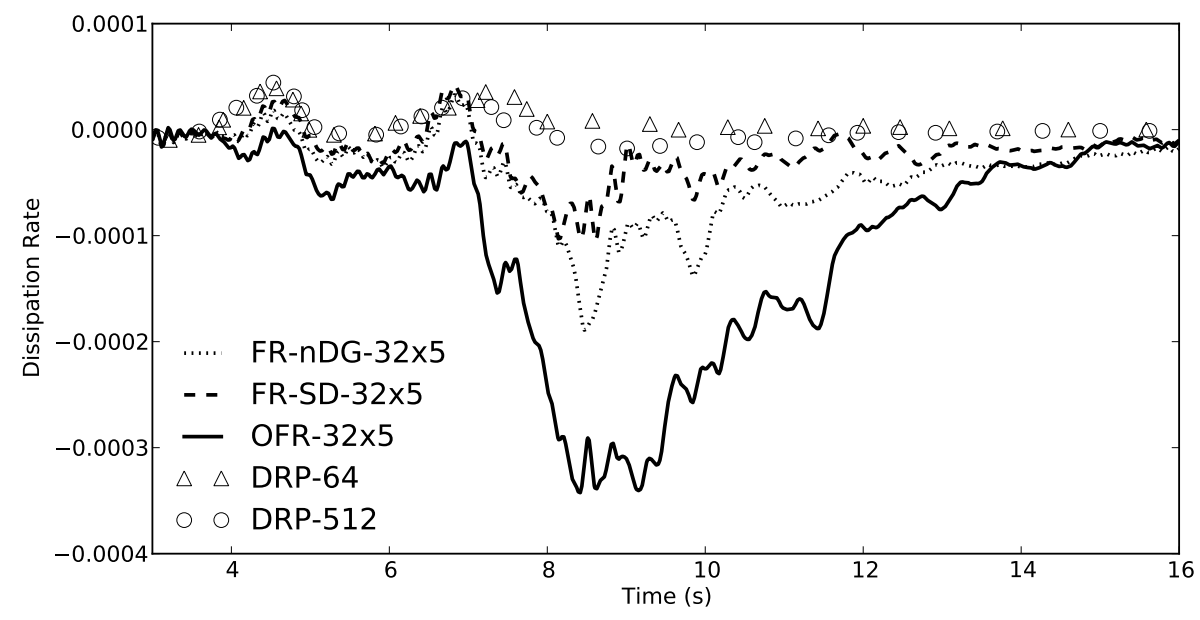

Fig. 8 Pressure dilatation-based dissipation rate $\epsilon_{5}$ using FR-nDG, FR-SD and OFR at $p_{4}$ on medium mesh. 'DRP-64'/'DRP-512' = DRP scheme on $64^{3} / 512^{3}$ DoF meshes [51].

\section{Energy Spectra}

Figure $9(\mathrm{a}-\mathrm{f})$ displays energy spectra of the $p_{3}(\mathrm{a}, \mathrm{b}), p_{4}(\mathrm{c}, \mathrm{d})$ and $p_{5}(\mathrm{e}, \mathrm{f})$ FR-nDG, FR$\mathrm{SD}$ and OFR solutions on the coarse and medium meshes at time $t=9$ seconds compared to the reference solution computed using a pseudo-spectral method on a $512^{3}$ mesh by Carton de Wiart et al. [37]. Note that the $p_{5}$ FR-SD scheme on the coarse mesh is not displayed because it diverged prior to $t=9$ seconds. The maximum resolvable wavenumber on each mesh/order pairing, given by $k_{\max }=D O F / 2$, is marked by a vertical dashed line. Significant discrepancies are observed at low wavenumbers between the coarse-mesh results and the reference solution, caused by poor representation of the flow dynamics at such low resolution. Similar effects were also observed by Beck and Gassner at low resolution [9]. The energy in the highest resolvable wavenumbers falls off short of $k_{\max }$ in the $p_{3}$ and $p_{4}$ spectra with all schemes, while at $p_{5}$ the spectra are much closer to the 


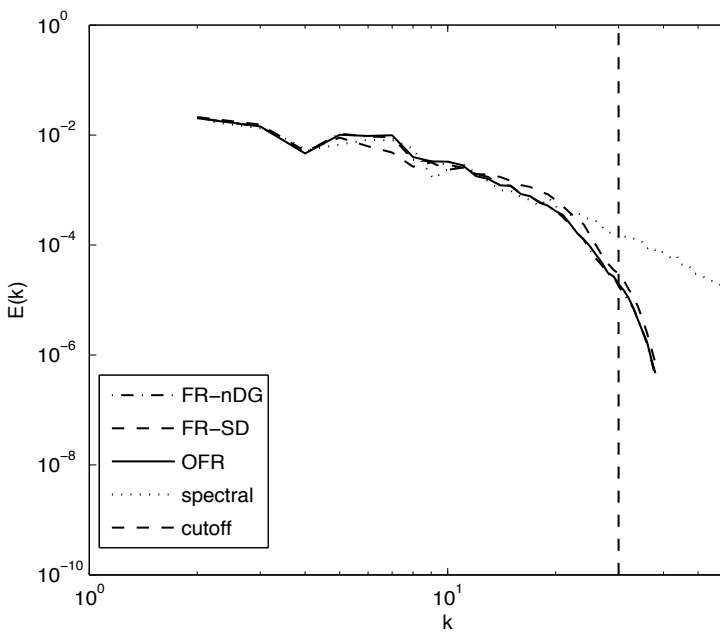

(a) $p_{3}$, coarse

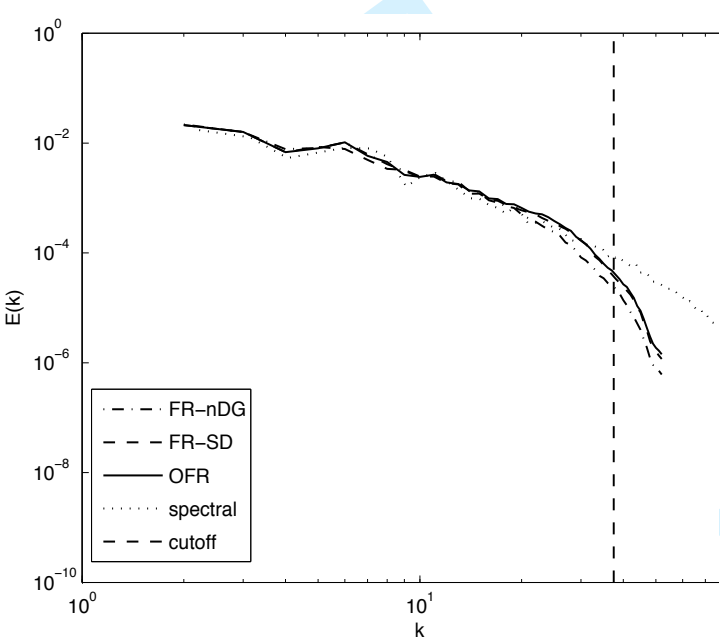

(c) $p_{4}$, coarse

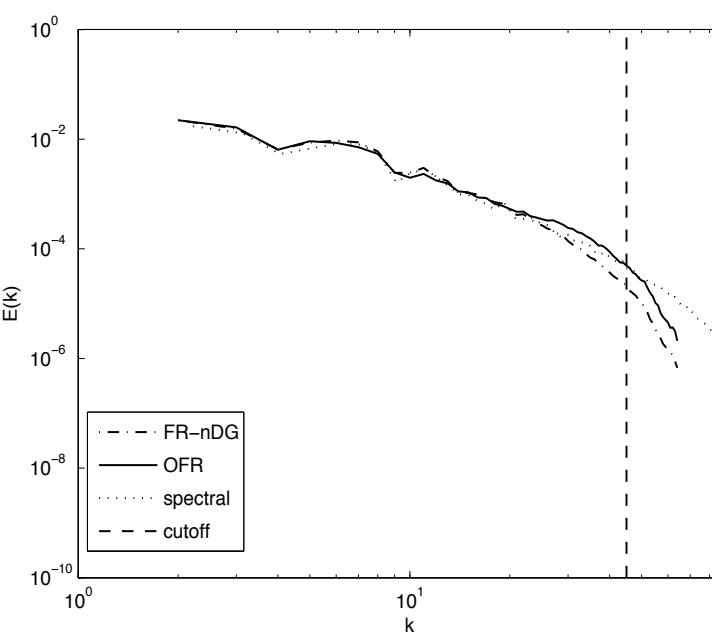

(e) $p_{5}$, coarse

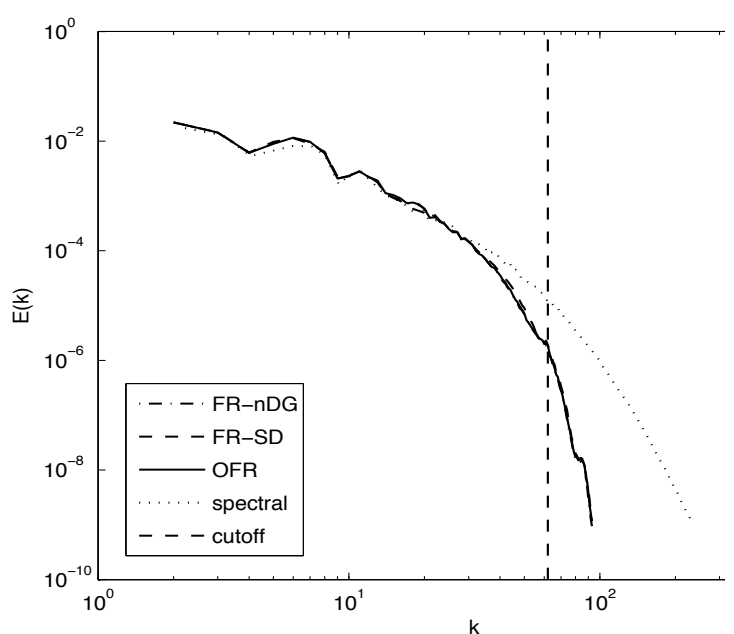

(b) $p_{3}$, medium

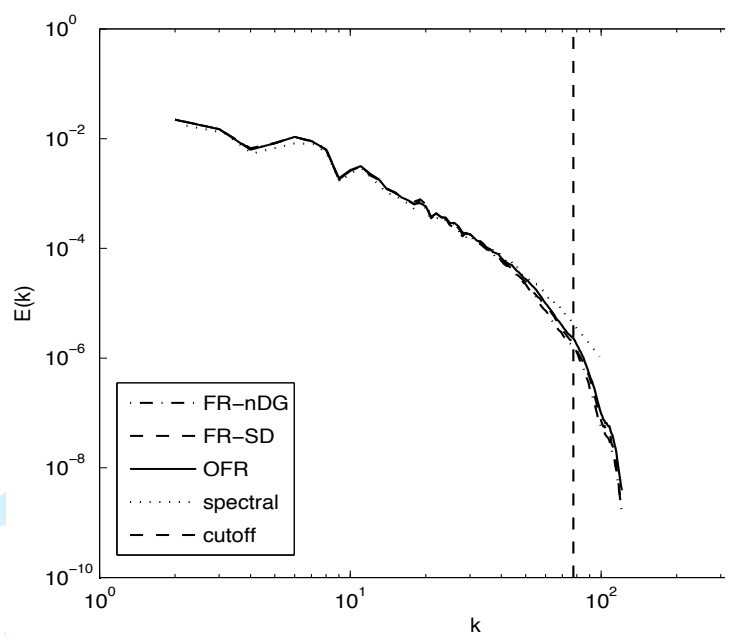

(d) $p_{4}$, medium

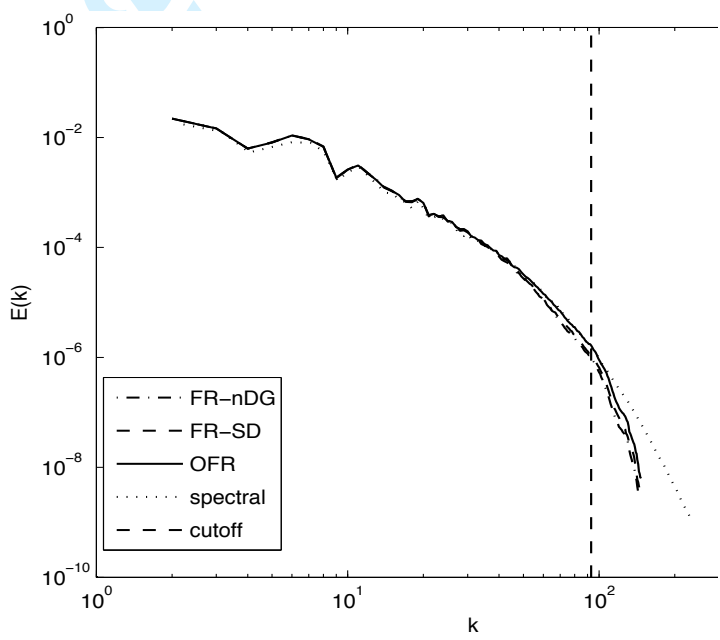

(f) $p_{5}$, medium

Fig. 9 Energy spectra of FR-nDG, FR-SD and OFR schemes at $t=9$ on coarse and medium meshes at $(\mathbf{a}, \mathbf{b}) p_{3},(\mathbf{c}, \mathbf{d}) p_{4}$ and $(\mathbf{e}, \mathbf{f}) p_{5}$ vs. reference spectral solution on $512^{3}$ DoF mesh [37]. 
reference solution. This suggests that higher polynomial orders are better at maintaining the energy content of the smaller scales on low-resolution meshes. Looking only at the medium-mesh results, at $p_{3}$ all the schemes produce similar spectra. As the order is increased, the differences between each scheme becomes more pronounced. The $p_{5}$ OFR spectra are much closer to the reference solution than the FR-nDG and FR-SD spectra; indeed the $p_{5}$ OFR spectrum on the medium mesh overlies the reference spectrum for most of the resolvable range of wavenumbers. It can be concluded that, as predicted by theory, the OFR scheme offers superior spectral resolution versus the FR-nDG and FR-SD schemes in practice, although the validity of this claim must also be verified in more complex test cases.

\section{Flow Visualization}

Figure 10 shows contours of instantaneous vorticity magnitude $|\boldsymbol{\omega}|$ on a small section of the plane defined by $x=0$ at time $t=8$, when the most complex vortical flow structures are generated. The reference data (solid black contours) was computed using a spectral element method with $512^{3}$ DoF by Carton de Wiart et al. [37] and is available from the 3rd High Order Workshop [40]. The solution obtained with the $p_{3}$ FR-nDG scheme on the fine mesh (filled contours with dashed divisions) is superimposed on the reference solution. Both sets of contours are defined by $|\boldsymbol{\omega}|=\{1,5,10,20,30\}$. The FR-nDG solution approximately overlies the reference solution but there are some discrepancies and noisy regions, possibly caused by the discontinuous nature of the solution and the relatively coarse mesh.

\section{Conclusion}

In this paper the performance of the Energy-Stable Flux Reconstruction (ESFR) schemes and the optimized OESFR/OFR schemes in simulations of under-resolved turbulent flow was investigated. The Taylor-Green Vortex problem at $R e=1600$ was simulated on a range of coarse meshes and polynomial orders using Energy-Stable Flux Reconstruction schemes encompassing the FRnDG, FR-SD, Optimized ESFR (OESFR) and Optimized FR (OFR) schemes. Predictions of the energy-based dissipation rate $\epsilon_{1}$ and vorticity-based dissipation rate $\epsilon_{2}$ showed that a balance must be struck between accuracy and stability. Holding the number of DoF constant, low-order $\left(p_{1}\right)$ 
Fig. 10 Contours of vorticity on the plane $x=0$ for $p_{3}$ FR-nDG on fine mesh (- -) and reference spectral solution with $512^{3}$ DoF (-) [37].

schemes on a fine mesh were inaccurate but stable due to high numerical dissipation, while highorder $\left(p_{7}\right)$ schemes on a coarse mesh were more accurate but unstable. Fourth-order accurate $\left(p_{3}\right)$ schemes offered a good balance of accuracy and stability; the $p_{3}$ FR-SD scheme on a mesh of $64^{3}$ elements (i.e., 256 DoF per direction) matched the reference solution. Instabilities in the solution at high orders are thought to originate from large variations in the high order polynomials within each element coupled with aliasing errors and accumulation of energy in the small scales. Further work is needed to develop effective nonlinear stabilization techniques such as filtering; current research in the Aerospace Computing Lab is directed at this purpose.

All the FR schemes tested were able to fairly accurately predict $\epsilon_{2}$ at orders of $p_{4}$ and above on a relatively coarse mesh, which is encouraging for their use in under-resolved simulations of high Reynolds number turbulent flows. The DG and OESFR schemes, which behaved an an identical manner in all tests, were the least accurate. The FR-SD scheme was slightly more accurate and the OFR scheme was the most accurate, achieving excellent agreement with the reference solution at $p_{6}$. The dissipation due compressibility effects, $\epsilon_{5}$, was over-predicted by all the FR schemes at 
low resolution. It appears likely that instabilities associated with high-order polynomials are at the root of the problem, which will the subject of future research. The energy spectra computed from the OFR results were in good agreement with the reference spectral solution of Carton de Wiart et al. [37] and were superior at high wavenumbers to the spectra of FR-nDG and FR-SD results. This demonstrates that the optimization of the FR correction functions for wave propagation by Asthana and Jameson [34] is effective when applied to the unsteady Navier-Stokes equations in three dimensions and motivates the future use of the OFR scheme for more complex turbulent flows. These results show that Flux Reconstruction is a very promising method for simulating turbulent flows on coarse meshes. More research is needed to increase stability at high orders by draining energy from the highest resolved wavenumbers. Future work will employ the FR schemes for LES of complex high Reynolds number flows.

\section{Acknowledgments}

This research was made possible by the support of the NSF under grant number 1114816, monitored by Dr Leland Jameson, and the Air Force Office of Scientific Research under grant number FA9550-10-1-0418, monitored by Dr Fariba Fahroo.

\section{References}

[1] Wang, Z., Fidkowski, K., Abgrall, R., Bassi, F., Caraeni, D., Cary, A., Deconinck, H., Hartmann, R., Hillewaert, K., Huynh, H., Kroll, N., May, G., Persson, P.-O., van Leer, B., and Visbal, M., "Highorder CFD methods: current status and perspective," Int. J. Num. Meth. Fl., Vol. 72, No. 8, 2013, pp. $811-845$ doi:10.1002/fld.3767.

[2] Lele, S., "Compact finite difference schemes with spectral-like resolution," J. Comput. Phys., Vol. 103, No. 1, 1992, pp. 16-42.

[3] Bassi, F. and Rebay, S., "A high-order accurate discontinuous finite element method for the numerical solution of the compressible Navier-Stokes equations," J. Comput. Phys., Vol. 131, No. 2, 1997, pp. $267-279$.

[4] Cockburn, B., Karniadakis, G., and Shu, C., Discontinuous Galerkin methods: theory, computation and applications, Springer, 2000. 
[5] Chapelier, J.-B., Plata, M., and Renac, F., "Inviscid and viscous simulations of the Taylor-Green vortex flow using a modal Discontinuous Galerkin approach," 42nd AIAA Fluid Dynamics Conference and Exhibit, 25 - 28 June 2012, New Orleans, Louisiana. AIAA paper 2012-3073.

[6] Birken, P., Gassner, G., Haas, M., and Munz, C., "Efficient time integration for discontinuous Galerkin method for the unsteady 3D Navier-Stokes equations," in Eberhardsteiner, J., ed., "ECCOMAS,", 2012.

[7] Hesthaven, J. and Warburton, T., Nodal discontinuous Galerkin methods: algorithms, analysis, and applications, Vol. 54, Springer, 2007.

[8] Kopriva, D., Implementing spectral methods for partial differential equations: algorithms for scientists and engineers, Springer, 1st ed., 2009.

[9] Beck, A. and Gassner, G., "On the accuracy of high-order discretizations for underresolved turbulence simulations," Theor. and Comp. Fluid Dyn., Vol. 27, No. 3-4, 2012, pp. 221-237, doi:10.1007/s00162-011-0253-7.

[10] Gassner, G., Lorcher, F., Munz, C., and Hesthaven, J., "Polymorphic nodal elements and their application in discontinuous Galerkin methods," J. Comput. Phys., Vol. 228, No. 5, 2009, pp. 1573-1590.

[11] Kopriva, D. A. and Kolias, J. H., "A conservative staggered-grid Chebyshev multidomain method for compressible flows," J. Comput. Phys., Vol. 125, 1996, pp. 244-261, doi:10.1006/jcph.1996.0091.

[12] Liu, Y., Vinokur, M., and Wang, Z. J., "Spectral difference method for unstructured grids I: basic formulation," J. Comput. Phys., Vol. 216, 2006, pp. 780-801, doi:10.1006/jcph.2002.7041.

[13] Huynh, H., "A flux reconstruction approach to high-order schemes including discontinuous Galerkin methods," 18th AIAA Computational Fluid Dynamics Conference, Miami, FL.

[14] Vincent, P. E., Castonguay, P., and Jameson, A., "A new class of high-order energy stable flux reconstruction schemes," J. Sci. Comput., Vol. 47, No. 1, 2011, pp. 50-72, doi:10.1007/s10915-010-9420-z.

[15] Castonguay, P., Williams, D., Vincent, P. E., and Jameson, A., "Energy stable flux reconstruction schemes for advection-diffusion problems," Computer Methods in Applied Mechanics and Engineering, Vol. 267, 2013, pp. 400-417, doi:10.1016/j.cma.2013.08.012.

[16] Williams, D. M., Castonguay, P., Vincent, P. E., and Jameson, A., "Energy stable flux reconstruction for advection-diffusion problems on rriangles," J. Comput. Phys., Vol. 250, 2012, pp. 53-76, doi:10.1007/s10915-010-9420-z. 
[17] Williams, D. M. and Jameson, A., "Energy stable flux reconstruction for advection-diffusion problems on tetrahedra," Journal of Scientific Computing, Vol. 59, 2013, pp. 721-759, doi:10.1007/s10915-013-9780-2.

[18] Jameson, A., Vincent, P. E., and Castonguay, P., "On the non-linear stability of flux reconstruction schemes," J. Sci. Comput., doi:10.1007/s10915-011-9490-6.

[19] Williams, D. and Jameson, A., "Nodal points and the nonlinear stability of high-order methods for unsteady flow problems on tetrahedral meshes," 43rd AIAA Fluid Dynamics Conference, June 24-27, 2013, San Diego, CA. AIAA Paper 2013-2830.

[20] Bogey, C. and Bailly, C., "Large eddy simulations of round free jets using explicit filtering with/without dynamic Smagorinsky model," International Journal of Heat and Fluid Flow, Vol. 27, 2006, pp. 603610 doi:10.1016/j.ijheatfluidflow.2006.02.008.

[21] Blackburn, H. and Schmidt, S., "Spectral element filtering techniques for large eddy simulation with dynamic estimation," J. Comput. Phys., Vol. 186, 2003, pp. 610-629, doi:10.1016/S0021-9991(03)00088-3.

[22] Karamanos, G. and Karniadakis, G., "A spectral vanishing viscosity method for large-eddy simulations," J. Comput. Phys., Vol. 163, No. 1, 2000, pp. 22-50, doi:10.1006/jcph.2000.6552.

[23] Pasquetti, R., "Spectral vanishing viscosity method for LES: sensitivity to the SVV control parameters," Journal of Turbulence, Vol. 6, No. 12, 2005, pp. 1-14, doi:10.1080/14685240500125476.

[24] Grinstein, F. and Fureby, C., "Recent progress on MILES for high Reynolds number flows," Journal of Fluids Engineering, Vol. 124, No. 4, 2002, pp. 848-861.

[25] Pasquetti, R., "High-order LES modeling of turbulent incompressible flow," Comptes Rendus Mécanique, Vol. 333, No. 1, 2005, pp. 39-49.

[26] Galbraith, M. and Visbal, M., "Implicit large eddy simulation of low Reynolds number flow past the SD7003 airfoil," Aiaa paper 2008-225, 46th Aerospace Science Meeting and Exhibit, Reno, Nv, 2008.

[27] Garmann, D., Visbal, M., and Orkwis, P., "Comparative study of implicit and subgrid-scale model large-eddy simulation techniques for low-Reynolds number airfoil applications," International Journal for Numerical Methods in Fluids, Vol. online only.

[28] Parsani, M., Ghorbaniasl, G., Lacor, C., and Turkel, E., "An implicit high-order spectral difference 
approach for large eddy simulation," J. Comput. Phys., Vol. 229, 2010, pp. 5373-5393.

[29] Diosady, L. and Murman, S., "Design of a variational multiscale method for turbulent compressible flows," in "21st AIAA Computational Fluid Dynamics Conference, San Diego, CA, June 24-27," , 2013.

[30] Roe, P. L., "Approximate Riemann solvers, parameter vectors and difference schemes," J. Comput. Phys., Vol. 43, 1981, pp. 357-372, doi:10.1016/0021-9991(81)90128-5.

[31] Jameson, A. and Lodato, G., "A note on the numerical dissipation from high-order discontinuous finite element schemes," Computers and Fluids, Vol. 98, 2013, pp. 186-195, doi:10.1016/j.compfluid.2014.01.016.

[32] Allaneau, Y. and Jameson, A., "Connections between the filtered discontinous Galerkin method and the flux reconstruction approach to high order discretizations," Computer Methods in Applied Mechanics and Engineering, pp. 3628-3636, doi:10.1016/j.cma.2011.08.01.

[33] Vincent, P. E., Castonguay, P., and Jameson, A., "Insights from von Neumann analysis of high-order flux reconstruction schemes," J. Comput. Phys., Vol. 230, No. 22, 2011, pp. 8134-8154.

[34] Asthana, K. and Jameson, A., "High-order flux reconstruction schemes with minimal dispersion and dissipation," J. Sci. Comp., pp. 1-32, doi:0.1007/s10915-014-9882-5. Published online 28 Jun. 2014.

[35] Don, W., Gottlieb, D., and Shu, C., "Numerical convergence study of nearly-incompressible, inviscid Taylor-Green vortex flow," J. Sci. Comput., Vol. 24, No. 1, 2005, pp. 1-27, doi:10.1007/s10915-004-5407-y.

[36] Johnsen, E., Varadan, S., and van Leer, B., "A three-dimensional recovery-based discontinuous Galerkin method for turbulence simulations," 51st AIAA Aerospace Sciences Meeting, 077 - 10 January 2013, Grapevine, Texas. AIAA Paper 2013-0515.

[37] Carton de Wiart, C., Hillewaert, K., Duponcheel, M., and Winckelmans, G. S., "Assessment of a discontinuous Galerkin method for the simulation of vortical flows at high Reynolds number," International Journal for Numerical Methods in Fluids, Vol. 74, 2014, pp. 469-493, doi:10.1002/fld.3859.

[38] "1st International Workshop on High-order CFD methods," At the 50th AIAA Aerospace Sciences Meeting, January 7-8 2012, Nashville, Tennessee, 2012.

[39] "2nd International Workshop on High-order CFD methods," At DLR, May 27-28, Cologne, Germany, 2013. 
[40] "3rd International Workshop on High-order CFD methods," At the 53rd AIAA Aerospace Sciences Meeting, Kissimmee, FL, 2015.

[41] Bull, J. and Jameson, A., "High-order flux reconstruction schemes for LES on tetrahedral meshes," in "Proceedings of the 5th Symposium on Hybrid RANS-LES Methods, College Station, TX," , 2014.

[42] López, M., Sheshadri, A., Bull, J., Economon, T., Romero, J., Watkins, J., Williams, D., Palacios, F., Jameson, A., and Manosalvas, D., "Verification and validation of HiFiLES: a high-order NavierStokes unstructured solver on multi-GPU platforms," 44th AIAA Fluid Dynamics Conference, Atlanta, Georgia, June 16-20, 2014.

[43] Rusanov, V. V., "Calculation of interaction of non-steady shock waves with obstacles," Journal of Computational Math and Physics USSR, Vol. 1, 1961, pp. 261-279.

[44] Kannan, R. and Wang, Z., "LDG2: A variant of the LDG flux formulation for the spectral volume method," Journal of Scientific Computing, Vol. 46, No. 2, 2011, pp. 314-328.

[45] Jameson, A., "A proof of the stability of the spectral difference method for all orders of accuracy," $J$. Sci. Comput., Vol. 45, No. 1, 2010, pp. 348-358, doi:10.1007/s10915-009-9339-4.

[46] Fischer, P. and Mullen, J., "Filter-based stabilization of spectral element methods," Comptes Rendus de l'Académie des Sciences-Series I-Mathematics, Vol. 332, No. 3, 2001, pp. 265-270, doi:10.1016/S0764-4442(00)01763-8.

[47] Castonguay, P., Liang, C., and Jameson, A., "Simulation of transitional flow over airfoils using the spectral difference method," in "AIAA Fluid Dynamics Conference and Exhibit, Chicago, Illinois, June 28 - July 1,", 2010.

[48] Ou, K. and Jameson, A., "towards computational flapping wing aerodynamics of realistic configurations using spectral difference method," 20th AIAA Computational Fluid Dynamics Conference, Honolulu, HI, June 27-30, 2011. AIAA Paper 2011-3068.

[49] Ou, K., Castonguay, P., and Jameson, A., "3D Flapping Wing Simulation with High Order Spectral Difference Method on Deformable Mesh," 49th AIAA Aerospace Sciences Meeting, Orlando, FL, Jan 4-7. AIAA Paper 2011-1316.

[50] Lodato, G., Castonguay, P., and Jameson, A., "Discrete filter operators for large-eddy simulation using high-order spectral difference methods," International Journal for Numerical Methods in Fluids, Vol. 72, No. 2, 2013, pp. 231-258, doi:10.1002/fld.3740.

[51] Debonis, J., "solutions of the Taylor-Green vortex problem using high-resolution explicit finite difference 
methods," 51st AIAA Aerospace Sciences Meeting, 07 - 10 January 2013, Grapevine, Texas. AIAA Paper 2013-0382.

[52] Bogey, C. and Bailly, C., "A family of low dispersive and low dissipative explicit schemes for flow and noise computations," J. Comput. Phys., Vol. 194, 2004, pp. 194-214, doi:10.1016/j.jcp.2003.09.003.

[53] Persson, P. and Peraire, J., "sub-cell shock capturing for discontinuous Galerkin methods," 44th AIAA Aerospace Sciences Meeting and Exhibit, Reno, NV, Jan 9-12, 2006. AIAA Paper 2006-112.

[54] Sheshadri, A. and Jameson, A., "Shock detection and capturing methods for high order discontinuousGalerkin finite element methods," AIAA Paper 2014-2688.

[55] Colonius, T. and Lele, S. K., "Computational aeroacoustics: progress on nonlinear problems of sound generation," Progress in Aerospace Sciences, Vol. 40, No. 6, 2004, pp. 345-416, doi:10.1016/j.paerosci.2004.09.001. 